\title{
Oxidative stress in asthmatic and non-asthmatic adolescent swimmers-A breathomics approach
}

\author{
Mariana Couto $^{1,2,3}$ (D) | Corália Barbosa ${ }^{4}$ | Diana Silva ${ }^{1,5}$ | Alisa Rudnitskaya ${ }^{6}$ | \\ Luís Delgado $^{1,3,5}$ | André Moreira ${ }^{1,5,7}$ | Sílvia M. Rocha4
}

${ }^{1}$ Basic and Clinical Immunology Unit, Department of Pathology, Faculty of Medicine, University of Porto, Porto, Portugal

${ }^{2}$ Immunoallergology, Hospital \& Instituto CUF Porto, Porto, Portugal

${ }^{3}$ CINTESIS, University of Porto, Porto, Portugal

${ }^{4}$ Department of Chemistry \&

QOPNA, University of Aveiro, Aveiro, Portugal

${ }^{5}$ Imunoalergologia, Centro Hospitalar São

João, EPE, Porto, Portugal

${ }^{6}$ Department of Chemistry \&

CESAM, University of Aveiro, Aveiro, Portugal

${ }^{7}$ EPIUnit Institute of Public Health, University of Porto, Porto, Portugal

Correspondence

Mariana Couto, Serviço e Laboratório

de Imunologia, Faculdade de Medicina,

Universidade do Porto, Porto, Portugal

Email: marianafercouto@gmail.com

Funding information

European Regional Development Fund FEDER through the Competitive Factors Thematic Operational Programme (COMPETE) and FCT, Portugal, under projects PEst-C/QUI/ UI0062/2013 (Research Unit 62/94 QOPNA) and PTDC/QUI-QUI/117803/2010.

\begin{abstract}
We hypothesize that oxidative stress induced by trichloramine exposure during swimming could be related to etiopathogenesis of asthma among elite swimmers.

Aim: To investigate the effect of a swimming training session on oxidative stress markers of asthmatic compared to non-asthmatic elite swimmers using exhaled breath (EB) metabolomics.
\end{abstract}

Methods: Elite swimmers annually screened in our department $(n=27)$ were invited and those who agreed to participate ( $n=20$, of which 9 with asthma) had EB collected (Tedlar ${ }^{\circledR}$ bags) before and after a swimming training session. SPME fiber (DVB/CAR/PDMS) was used to extract EB metabolites followed by a multidimensional gas chromatography analysis (GC ×GC-ToFMS). Dataset comprises eight metabolites end products of lipid peroxidation: five aliphatic alkanes (nonane, 2,2,4,6,6-pentamethylheptane, decane, dodecane, and tetradecane) and three aldehydes (nonanal, decanal, and dodecanal). To assess exercise impact on lipid peroxidation markers, data were analyzed using principle component analysis (PCA), which was run on the original data set and on the data set constructed using differences in the metabolite total areas before and after exercise session.

Results: Heatmap representation revealed that metabolites content decreased after exercise, both for control and asthma groups; however, the greater decrease was observed for controls. Asthmatics and controls did not form separated clusters; however, control swimmers demonstrated a more varied response to the exercise being dispersed along all score plot.

Conclusion: In well-trained athletes, swimming is associated with a decrease in oxidative stress markers independently of the presence of asthma, although a more pronounced decrease was seen in controls.

\section{KEYWORDS}

adolescents, asthma, breathomics, exercise, exhaled breath, lipid peroxidation, metabolomic breath analysis, oxidative stress, swimmers

\section{BACKGROUND}

The high prevalence of asthma among elite athletes has been related to bronchial epithelial damage. Oxidative stress is believed to contribute to the pathophysiology of asthma and, together with increased ventilation induced by exercise, may be a final common pathway leading to tissue damage. ${ }^{1}$ Swimmers are among the athletes with higher prevalence of asthma and present an increased risk for 
a specific phenotype, ${ }^{2}$ but the cause for it remains under debate. Trichloramine exposure in swimming pools may result in oxidative stress through reaction with substrates in the epithelial lining, therefore decreasing bronchial intrinsic antioxidants and producing subsequent inflammation, progression of bronchial hyper-responsiveness, and a vicious cycle of continued airway oxidative stress and inflammation. ${ }^{3}$ Therefore, we hypothesized that exposure to chloramines during swimming would induce oxidative stress, which could be related to the etiopathogenesis of asthma in swimmers. In an exploratory study using metabolomic analysis of exhaled breath (EB) of elite swimmers, we aimed to assess the effect of a swimming training session on oxidative stress lipid peroxidation markers and to investigate whether the impact is similar across asthmatic and non-asthmatic subjects.

\section{2 | METHODS}

The participants included in this observational study have been previously described. ${ }^{4}$ Data from the screening visits performed the beginning of the training season were retrospectively collected (skin prick tests with aeroallergens, spirometry, bronchodilation (BD), and airway responsiveness to methacholine). In these visits, swimmers were asked to withhold anti-asthmatic and/or anti-allergic medication. ${ }^{5}$ Demographic data, respiratory parameters, and skink prick tests results are presented as supplementary files (Table S1 and Table S2). No significant differences were observed between asthmatic and non-asthmatic swimmers for demographic and personal characteristics, except for the expected lower $\mathrm{PD}_{20}$ among those with asthma. ${ }^{4}$

The 20 swimmers who agreed to this study (nine had asthma according to the International Olympic Committee criteria ${ }^{6}$ ) had a specific evaluation collecting EB before and 5 minutes after a training session at their swimming pool. This evaluation was performed during summer, and two to three swimmers had EB collected in each visit (a total of eight investigators' visits of the over 2 weeks). Swimmers performed their regular 1-hour training in an open-air chlorine disinfected swimming pool, and no changes on the training session (swimming style and the intensity of the training-Table S1) were imposed by the investigators.

Written informed consent and ethical commission approval were obtained.

\section{1 | Procedures}

Airway responsiveness to methacholine was measured using a fivebreath dosimeter protocol with quadrupling doses (MEFAR MB3), starting with a $0.0625 \mathrm{mg} / \mathrm{mL}$ solution. ${ }^{5}$ Spirometry was performed according to guidelines. ${ }^{7}$ Results of spirometry $\left(\mathrm{FEV}_{1}, \mathrm{FVC} \mathrm{FEF}_{25-75}\right)$ are presented as absolute and predicted values, according to reference algorithms. ${ }^{8}$ Positive BD was defined as an increase in $\mathrm{FEV}_{1} \geq 12 \%$ and $200 \mathrm{~mL}$, after 15 minutes of $400 \mu \mathrm{g}$ inhaled salbutamol. ${ }^{9}$ Skin prick tests to common aeroallergens were performed (Leti ${ }^{\circledR}$ ) (Table S2), defining atopy as the presence of at least one positive $(\geq 3 \mathrm{~mm})$ result. A heart rate monitor watch was used to measure heart rate.
EB was collected in 1-L Tedlar ${ }^{\circledR}$ bags (SKC Inc., Eighty Four, PA, USA), and two samples were collected for each swimmer (one before and other after a training session). Before collecting EB, all bags were thoroughly cleaned to remove residual contaminants by flushing with high purity nitrogen gas. The breath sampling parameters were previously optimized. ${ }^{10}$ Briefly, at each sampling moment, the swimmer was asked to cleanse mouth with water before sampling and subsequently instructed to inhale and exhaled normally and then exhale deeply using a disposable mouthpiece into the Tedlar ${ }^{\circledR}$ bag previously holding the breath for 5 seconds. The solid-phase microextraction (SPME) fiber (DVB/CAR/PDMS-50/30 $\mu \mathrm{m}$ divinylbenzene/carboxen $^{\mathrm{Tm}} /$ poly (dimethylsiloxane StableFlex ${ }^{\mathrm{Tm}} 1 \mathrm{~cm}$ ) was used to extract breath metabolites from Tedlar $^{\circledR}$ bag. The bags were stored at $22^{\circ} \mathrm{C}$ and analyzed in a maximum of 6 hours, as recommended. ${ }^{11}$ The SPME fiber with sorbed metabolites was introduced into the GC×GC-ToFMS (comprehensive two-dimensional gas chromatography combined with time of flight mass spectrometry detection) injection port as reported by Caldeira et al. ${ }^{12}$ Each breath represented a single sample, being analyzed once. A LECO Pegasus 4D (LECO, St. Joseph, MI, USA) GC ×GCToFMS system was used, which consist of an Agilent GC 7890A gas chromatograph, with a dual stage jet cryogenic modulator (licensed from Zoex) and a secondary oven. The detector is a high-speed ToF mass spectrometer. The instrumental parameters for breath analysis were previously established. ${ }^{12}$ For identification purposes, the mass spectrum of each selected metabolite was compared to those in mass spectral libraries of one home-made (using standards) and two commercial databases (Wiley 275 and US National Institute of Science and Technology (NIST) V.2.0-Mainlib and Replib). The identification is also supported by experimentally determining the RI (retention index) ${ }^{13}$ and standards co-injection (Table S3). The DTIC (Deconvoluted Total Ion Current) $\mathrm{GC} \times \mathrm{GC}$ area data were used as an approach to estimate the relative content of each metabolite in the EB from each subject and collection time.

\section{2 | Data analysis}

From several hundreds of metabolites detected on EB, a specific metabolite data set was established for a fast access of information from EB metabolomic profile, reducing the time of data pre-processing (from raw instrumental data to clean data for statistical processing) and thus becoming more expedite method for clinical purposes. Five aliphatic alkanes (nonane, 2,2,4,6,6-pentamethylheptane, decane, dodecane, and tetradecane) and three aldehydes (nonanal, decanal, and dodecanal), which may be used to assess lipid peroxidation state, were selected based on previous data. ${ }^{12}$

For comparison of before and after exercise session, peak areas of the selected aliphatic aldehydes and alkanes were extracted from the chromatograms and used to build the data matrix. This matrix consisted of 40 observations (breath samples of 20 individuals, with two observations from each subject-before and after exercise) and eight variables (peak areas of selected metabolites-Table S3). Data set was normalized; that is, variables were mean centered and divided by their standard deviations, prior to all calculations. To assess exercise 
impact on lipid peroxidation markers principle component analysis (PCA), an unsupervised tool was used, which was run on the data set constructed using differences in the metabolite total areas before and after exercise session. In addition, a heatmap visualization has been performed on the data from control and asthma groups before and after exercise. Calculations were performed using MATLAB (MATrix LABoratory) v.7.3.0.

\section{3 | RESULTS}

Specific changes of each selected metabolite for each swimmer, before and after the exercise session, are shown in a heatmap representation (Figure 1), separated by asthma and control groups. Each metabolite was illustrated through different colors, corresponding dark blue and dark red colors to the lower and higher intensities, respectively. Heatmap representation revealed that generally the metabolites content decreased after exercise, both for control and asthma groups; however, the greater decrease was observed for controls.

The impact of the swimming session on the athletes was individualized, as illustrated by the scores and loadings bi-plot (Figure 2) produced using differences in metabolite concentrations after and before the exercise session. Swimmers were dispersed through principle component 1 (PC1), that explains $34 \%$ of the data variability, being the controls closer to PC1 negative and origin (swimmers that had no or very small changes in the metabolites content before and after exercise session are situated close to origin). The asthmatics are dispersed through PC1 positive more close to the metabolites position. Moreover, non-asthmatic atopic subjects seem to be located closer to those with asthma in the PCA score plot. Combining results from Figures 1 and 2, it should be highlighted that asthma and control groups did not form separated clusters with respect to studied metabolites. However, controls demonstrated a more varied response to the exercise being dispersed along all score plot (Figure 2), while asthmatics formed a more compact group located closer to the origin.

\section{4 | DISCUSSION}

Our results show that oxidative stress markers decrease after a swimming session both for control and asthmatic elite swimmers. There are possible explanations. These are well-trained athletes and, although acute physical exercise performed at high intensity may result in oxidative stress due to reactive oxygen species (ROS) being generated excessively by enhanced oxygen consumption ${ }^{14}$ (known as exerciseinduced oxidative stress), several studies have demonstrated that continuous aerobic training reduces ROS production and increases antioxidant defenses. ${ }^{14-17}$ Exercise training may compensate oxidative stress by adaptation of the antioxidant and repair systems; this may result in a decreased resting level of oxidative damage and an increased resistance to oxidative stress. ${ }^{14,18-20}$ Increased levels of ROS and oxidative damage are initiators of specific adaptive responses, such as the activation of antioxidant enzymes ${ }^{21}$ and enhanced oxidative damage repair. ${ }^{19}$ On the other hand, we cannot exclude the hypothesis of the oxidative stress markers reduction being related to changes in bronchial diameter: Exercise leads to increased ventilation and reduced airway caliber in asthmatic subjects, increasing airflow velocity; the reduced airway surface area may result in a lower diffusion of the metabolites through the airways. Also, EB was collected shortly after exercise, so the decrease observed in the oxidative stress markers may be due to washout of markers in EB given the high minute ventilation, as occurs with exhaled nitric oxide levels after forced breathing maneuvers. Besides, the usefulness of alkanes has been demonstrated to distinguish children with allergic asthma from healthy controls, ${ }^{12}$ which is not the case for many swimmers that present a specific phenotype of asthma not related to atopy. ${ }^{2}$

Swimmers with and without asthma did not aggregate into separated clusters with respect to studied metabolites; however, a more pronounced decrease was observed for controls, raising the possibility of a parallel production of these compounds in asthmatic swimmers. These findings suggest that a possible relation between asthma and oxidative stress could not be ruled out. Activated inflammatory cells in the airways of asthmatic subjects may be responsible for the

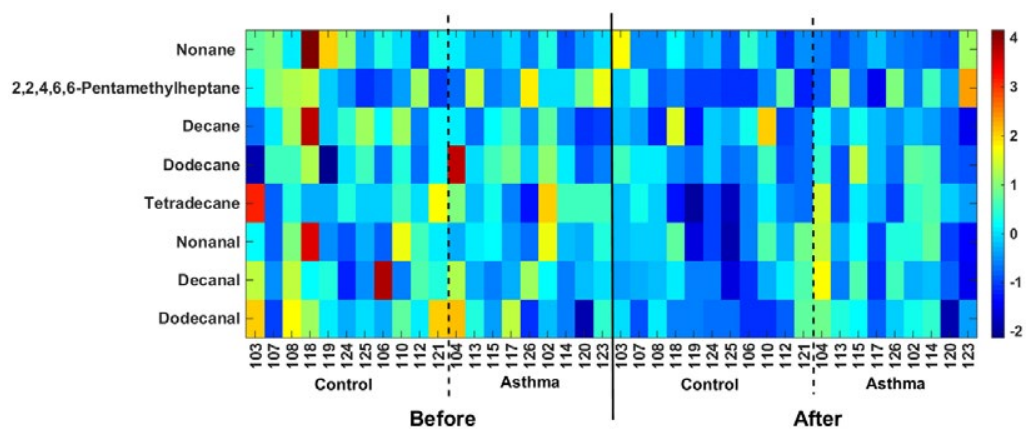

FIGURE 1 Heatmap representation of selected aliphatic alkanes and aldehydes level of each control and asthma subjects before and after exercise session. Each line corresponds to one metabolite (organized by aldehydes and alkanes chemical families), and each column corresponds to each individual (see individual number assignment in Table S1). Each compound was illustrated through different colors, allowing a visual assessment of the relative abundance of each one: Dark blue and dark red colors correspond to the fewer and larger amounts, respectively. [Colour figure can be viewed at wileyonlinelibrary.com] 


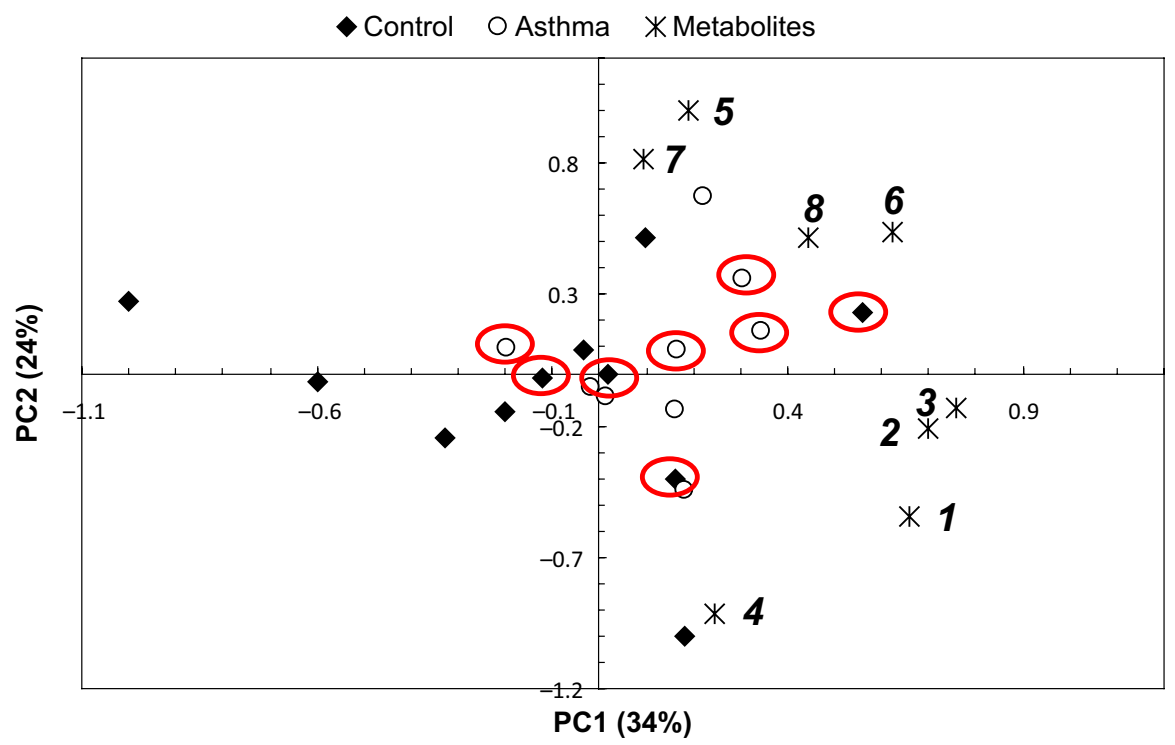

FIGURE 2 Principal component analysis (PCA) score and loading biplot. PCA scores and loadings were calculated using differences between concentrations of eight metabolites (see peak assignment in Table S3, supplementary file) in the exhaled breath before and after exercise session of control (full symbols) and asthma (open symbols) individuals. First and second principal components (PC1×PC2) explain 58\% of the data variability, being samples dispersed according PC1. Metabolite concentrations were calculated as GC $\times$ GC peak areas of selected aliphatic aldehydes and alkanes. Swimmers that had no or very small alteration in the metabolites content before and after exercise session are situated close to origin. Red circles were used to highlight the atopic individuals' position in the PCA biplot. [Colour figure can be viewed at wileyonlinelibrary.com]

biosynthesis of these metabolites. The production of aldehydes and alkanes by asthmatic subjects possibly turns them less prone to evidence such a marked decrease in oxidative stress metabolites as controls do. Exposure to environmental irritants leads to the recruitment and activation of inflammatory cells in asthmatic airways. Activated inflammatory cells respond with a "respiratory burst" that involves the uptake of oxygen and subsequent release of ROS into surrounding cells, ${ }^{1}$ which can damage lung tissue, contributing to oxidative stress in asthma. Oxidative stress can have many detrimental effects on airway function, including airway smooth muscle contraction, airway hyper-responsiveness, mucus hypersecretion, epithelial shedding, and vascular exudation ${ }^{1}{ }^{22}$ Under oxidative stress condition, damaged, dead, or activated structural cells can release damage-associated molecular patterns (DAMPs) and cytokines, able to promote dendritic cells maturation. ${ }^{23}$ In agreement, it has been recently shown that in competitive swimmers, the intensive training combined with exposure to by-products of chlorination induces airway epithelial damage and is associated with increased DAMPs, innate cytokine release, and neutrophilic airway inflammation. ${ }^{24}$ Moreover, non-asthmatic atopic subjects seem to be located closer to those with asthma in the PCA score plot, which is also in agreement with recent studies showing that inadequate antioxidant responses are strongly associated with allergic sensitization. ${ }^{23}$ It has been hypothesized that oxidative stress occurs in many allergic and immunologic disorders as a result of the imbalance between the endogenous production of free ROS and/or the reduction in antioxidant defense mechanisms. About half the asthmatic swimmers were atopic, but meanwhile, also $67 \%$ were on inhaled corticosteroids at the time of EB collection, which could reverse the production of lipid peroxidation metabolites biosynthesis by the inflammatory cells. More studies with larger samples are needed to clarify these differences.

Some swimmers presented slight changes in the metabolite concentrations while other had large changes, and such differences were not explained by the presence of asthma or atopy. During exercise, the rate of lipid oxidation is influenced by a number of factors influencing different sites in the metabolic pathway, which could be responsible for the differences observed. Some of these factors are related to the system/machinery and others to the specific conditions prevailing during exercise. We investigated the possible role of some of these factors including the activity of both the parasympathetic and the sympathetic nervous systems, exercise intensity, exercise duration, subject's temperature, sex, age, height, weight, body mass index, methacholine $\mathrm{PD}_{20}$, respiratory parameters, and the duration of lifetime years of competition, and we failed to observe any differences that could explain the distinct changes in metabolites across swimmers (data not shown). Nonetheless, there are several other possible explanatory factors that were not approached and need to be addressed in further studies.

There are some limitations in our study. First, EB was collected right after exercise and, if repeated later, possible washout effects induced by increased ventilation could have been avoided. Second, due to the inclusion of only elite swimmers from the same team in order to homogenize the characteristics of training and environment, we cannot assure that lack of differences between asthmatic and healthy swimmers are not related to low power to detect it. Third, we did not assess lung function after swimming, which could have provided some details about the occurrence of EIB. Fourth, the lack of a control group without swimming but exposed to chlorinated water disables the possibility 
to distinguish the effect of exercise from that of trichloramine exposure. Also, we have used an indirect measure of oxidative stress, for example, alkanes and aldehydes. However, most of the current literature uses indirect detection of ROS intervention, based on the dosage of specific end products resulting from the interaction of the ROS with biological macromolecules, such as lipids. The appearance of these end products, mainly alkanes, serves as proof of the prior existence of ROS. Finally, swimmers were practicing in an outdoor swimming pool, which could have reduced the exposure to chloramines. Nevertheless, competitive swimmers aspirate water droplets and chemicals when breathing the air floating just above the water surface and it has been reported that a significant increase in asthma occurs with the lifetime number of hours spent also in outdoor pools. ${ }^{25}$

Research in this area should be further developed. Our study should be followed by others with more athletes, training in indoor environments, and using repeated samples in different point times after exercise. Although EB has numerous advantages over other substrates, employing different substrates such as urine or plasma, rather than only EB, will support these results.

This is the first study using a metabolomic analysis of EB in elite swimmers to analyze the impact of an exercise challenge in oxidative stress. It has been advocated that challenging metabolic regulatory processes by testing the individual capacity and flexibility to cope with environmental stressors may unmask early changes in metabolism. ${ }^{26}$ Nevertheless, most metabolomics studies to date are limited to crosssectional analysis, and only few report time-resolved metabolome changes in response to a challenge. ${ }^{26}$ The present study contributes to overcome this paucity of data as infield metabolic analyses may be relevant to understand, support, and train elite athletes, highlighting the concept of sportomics. ${ }^{27}$

\section{ACKNOWLEDGMENTS}

The authors would like to thank all participants and the technical and administrative staff of FC Porto Swimming Section for logistical help. We also acknowledge Carla Martins (Centro Hospitalar São João, EPE) for help in methacholine bronchial provocation challenges, Diater ${ }^{\circledR}$ for providing methacholine, and Samuel Queirós and Paulo Santos (Faculty of Medicine, University of Porto) for the help in data collection. Project NORTE-01-0145-FEDER-000010-Health, Comfort and Energy in the Built Environment (HEBE), cofinanced by Programa Operacional Regional do Norte (NORTE2020), through Fundo Europeu de Desenvolvimento Regional (FEDER).

\section{REFERENCES}

1. Wood LG, Gibson PG, Garg ML. Biomarkers of lipid peroxidation, airway inflammation and asthma. Eur Respir J. 2003;21:177-186.

2. Couto M, Stang J, Horta L, et al. Two distinct phenotypes of asthma in elite athletes identified by latent class analysis. J Asthma. 2015;52:897-904.

3. Fisk MZ, Steigerwald MD, Smoliga JM, Rundell KW. Asthma in swimmers: a review of the current literature. Phys Sportsmed. 2010;38:28-34.
4. Couto M, Santos P, Silva D, Delgado L, Moreira A. Exhaled breath temperature in elite swimmers: the effects of a training session in adolescents with or without asthma. Pediatr Allergy Immunol. 2015;26:564-570.

5. Crapo RO, Casaburi R, Coates AL, et al. Guidelines for methacholine and exercise challenge testing-1999. This official statement of the American Thoracic Society was adopted by the ATS Board of Directors, July 1999. Am J Respir Crit Care Med. 2000;161: 309-329.

6. Carlsen $\mathrm{K}-\mathrm{H}$, Anderson S, Bjermer L, et al. Exercise-induced asthma, respiratory and allergic disorders in elite athletes: epidemiology, mechanisms and diagnosis: part I of the report from the Joint Task Force of the European Respiratory Society (ERS) and the European Academy of Allergy and Clinical Immunology (EAACI) in cooperation with GA2LEN. Allergy. 2008;63:387-403.

7. Miller MR, Hankinson J, Brusasco V, et al. Standardisation of spirometry. Eur Respir J. 2005;26:319-338.

8. Stanojevic S, Wade A, Stocks J, et al. Reference ranges for spirometry across all ages: a new approach. Am J Respir Crit Care Med. 2008;177:253-260.

9. Pellegrino R, Viegi G, Brusasco V, et al. Interpretative strategies for lung function tests. Eur Respir J. 2005;26:948-968.

10. Caldeira M, Barros AS, Bilelo MJ, Parada A, Câmara JS, Rocha SM. Profiling allergic asthma volatile metabolic patterns using a headspace-solid phase microextraction/gas chromatography based methodology. J Chromatogr A. 2011;1218:3771-3780.

11. Mochalski P, Wzorek B, Sliwka I, Amann A. Suitability of different polymer bags for storage of volatile sulphur compounds relevant to breath analysis. J Chromatogr B. 2009;877:189.

12. Caldeira M, Perestrelo R, Barros AS, et al. Allergic asthma exhaled breath metabolome: a challenge for comprehensive two-dimensional gas chromatography. J Chromatogr A. 2012;1254:87-97.

13. Rocha SM, Caldeira M, Carrola J, Santos M, Cruz N, Duarte IF. Exploring the human urine metabolomic potentialities by comprehensive two-dimensional gas chromatography coupled to time of flight mass spectrometry. J Chromatogr A. 2012;1252:1 55-163.

14. Vezzoli A, Pugliese L, Marzorati M, Serpiello FR, La Torre A, Porcelli S Time-Course Changes of Oxidative Stress Response to High-Intensity Discontinuous Training versus Moderate-Intensity Continuous Training in Masters Runners. PLoS ONE. 2014;9:e87506.

15. Miyazaki H, Oh-ishi S, Ookawara T, et al. Strenuous endurance training in humans reduces oxidative stress following exhausting exercise. Eur J Appl Physiol. 2001:84:1-6.

16. Fatouros IG, Jamurtas AZ, Villiotou V, et al. Oxidative stress responses in older men during endurance training and detraining. Med Sci Sports Exerc. 2004;36:2065-2072.

17. Powers SK, Ji LL, Leeuwenburgh C. Exercise training-induced alterations in skeletal muscle antioxidant capacity: a brief review. Med Sci Sports Exerc. 1999;31:987-997.

18. Alessio HM, Goldfarb AH. Lipid peroxidation and scavenger enzymes during exercise: adaptive response to training. J Appl Physiol (1985). 1988;64:1333-1336.

19. Radák Z, Kaneko T, Tahara S, et al. The effect of exercise training on oxidative damage of lipids, proteins, and DNA in rat skeletal muscle: evidence for beneficial outcomes. Free Radic Biol Med. 1999;27: 69-74.

20. Radak Z, Chung HY, Goto S. Systemic adaptation to oxidative challenge induced by regular exercise. Free Radic Biol Med. 2008;44:153-159.

21. Ji LL, Gomez-Cabrera MC, Steinhafel N, Vina J. Acute exercise activates nuclear factor (NF)-kappaB signaling pathway in rat skeletal muscle. FASEB J. 2004;18:1499-1506.

22. Biagioli MC, Kaul P, Singh I, Turner RB. The role of oxidative stress in rhinovirus induced elaboration of IL-8 by respiratory epithelial cells. Free Radic Biol Med. 1999;26:454-462. 
23. Utsch L, Folisi C, Akkerdaas JH, et al. Allergic sensitization is associated with inadequate anti-oxidant responses in mice and men. Allergy. 2015;70:1246-1258.

24. Seys SF, Hox V, Van Gerven L, et al. Damage-associated molecular pattern and innate cytokine release in the airways of competitive swimmers. Allergy. 2015;70:187-194.

25. Bernard A, Nickmilder M, Voisin C. Outdoor swimming pools and the risks of asthma and allergies during adolescence. Eur Respir J. 2008;32:979-988.

26. Krug S, Kastenmüller G, Stückler F, et al. The dynamic range of the human metabolome revealed by challenges. FASEB J. 2012;26:2607-2619.

27. Bassini A, Cameron LC. Sportomics: building a new concept in metabolic studies and exercise science. Biochem Biophys Res Commun. 2014;445:708-716.

\section{SUPPORTING INFORMATION}

Additional Supporting Information may be found online in the supporting information tab for this article.

How to cite this article: Couto M, Barbosa C, Silva D, et al. Oxidative stress in asthmatic and non-asthmatic adolescent swimmers-A breathomics approach. Pediatr Allergy Immunol. 2017;28:452-457. https://doi.org/10.1111/pai.12729 